\title{
An $A C$ modulated Near InfraRed gain calibration system for a 'Violin-Mode' transimpedance amplifier, intended for advanced LIGO suspensions
}

\author{
N.A. Lockerbie and K.V. Tokmakov \\ SUPA (Scottish Universities Physics Alliance) Department of Physics, \\ University of Strathclyde, 107 Rottenrow, Glasgow G4 0NG, UK.
}

\begin{abstract}
The background to this work was a prototype shadow sensor, which was designed for retro-fitting to an Advanced LIGO (Laser Interferometer Gravitational wave Observatory) testmass/mirror suspension, in which a $40 \mathrm{~kg}$ test-mass/mirror is suspended by four approximately $600 \mathrm{~mm}$ long by $0.4 \mathrm{~mm}$ diameter fused-silica suspension fibres. The shadow sensor comprised a LED source of Near InfraRed (NIR) radiation, and a 'tall-thin' rectangular silicon photodiode detector, which together were to bracket the fibre under test. The photodiode was positioned so as to be sensitive (primarily) to transverse 'Violin-Mode' vibrations of such a fibre, via the oscillatory movement of the shadow cast by the fibre, as this moved across the face of the detector. In this prototype shadow sensing system the photodiode was interfaced to a purposebuilt transimpedance amplifier, this having both $A C$ and $D C$ outputs. A quasi-static calibration was made of the sensor's $D C$ responsivity, i.e., incremental rate of change of output voltage versus fibre position, by slowly scanning a fused-silica fibre sample transversely through the illuminating beam. The work reported here concerns the determination of the sensor's more important AC (Violin-Mode) responsivity. Recognition of the correspondence between direct $A C$ modulation of the source, and actual Violin-Mode signals, and of the transformative rôle of the $A C / D C$ gain ratio for the amplifier, at any modulation frequency, $f$, resulted in the construction of the $A C / D C$ calibration source described here. A method for determining in practice the transimpedance $A C / D C$ gain ratio of the photodiode and amplifier, using this source, is illustrated by a specific numerical example, and the gain ratio for the prototype sensing system is reported over the frequency range $1 \mathrm{~Hz}-300 \mathrm{kHz}$. In fact, a maximum $D C$ responsivity of $1.26 \mathrm{kV} . \mathrm{m}^{-1}$ was measured using the prototype photodiode sensor and amplifier discussed here. Therefore, the measured AC/DC transimpedance gain ratio of 922.5 for this sensor, at $500 \mathrm{~Hz}$, translated into a maximum Violin-Mode $(A C)$ responsivity of $(1.16 \pm 0.05)$ $\mathrm{MVm}^{-1}$, at that frequency.
\end{abstract}

PACS numbers: 04.80.Nn, 84.30.-r, 06.30.Bp, 07.07.Df, 07.57.-c

\section{Introduction}

A prototype system of four shadow-sensors was designed to be retro-fitted to an advanced LIGO (Laser Interferometer Gravitational wave Observatory) test-mass/mirror suspension, in which a $40 \mathrm{~kg}$ test-mass is suspended by four fused silica fibres, the dimensions of the fibres being approximately $600 \mathrm{~mm}$ long by $0.4 \mathrm{~mm}$ in diameter [1-6]. Initially, the shadow-sensor - one intended for each suspension fibre-consisted of a single 'tall, narrow' rectangular silicon photodiode (Hamamatsu S2551, [7]), together with a collimated source of Near InfraRed (NIR: $\lambda=880-890 \mathrm{~nm}$ ) illumination-this casting a shadow of the illuminated fibre over one vertical edge the facing detector. In later work, however, a higher displacement sensitivity (but more elaborate) 'synthesized split-photodiode' detector was employed [8,9]. The principal purpose of the full detection system, using either detector, was to monitor any lateral 'Violin-Mode' resonances that might be excited in these fibres. The purpose-built Violin-Mode (VM) amplifier, which was interfaced to the detector, had two separate outputs: a relatively low (transimpedance) gain ' $D C$ ' output, to monitor the photodiode's quiescent photocurrent, and a much higher transimpedance gain, but lower displacement-sensing range, $A C$ output, this being intended for detecting $V M$ oscillations - ultimately, down to below 100 picometres (rms), in 1 second, at frequencies in the range $500 \mathrm{~Hz}-5 \mathrm{kHz}[8,9,11]$. Sensing $V M$ oscillations was the primary function of 
the shadow-sensor, but an important ancillary rôle of the amplifier's separate $D C$ and $A C$ outputs was to allow an actual calibration of the $V M(A C)$ displacement responsivity of the detection system to be made. This calibration was accomplished in two stages.

Firstly, a $0.4 \mathrm{~mm}$ diameter silica-fibre sample was translated at a steady rate $\left(\sim 50 \mu \mathrm{m} \mathrm{s}{ }^{-1}\right)$ transversely through the shadow-sensor's illuminating NIR beam, and the resulting 'notch' was recorded in the amplifier's $D C$ output voltage as a function of fibre position-the notch occurring as the fibre's shadow passed across the face of the photodiode detector. The shadow-notch voltage then was differentiated off-line, as a function of fibre position, so as to yield the rate-of-change of detected $D C$ voltage with incremental fibre displacement, versus mean fibre position. This quantity was the $D C$ (quasi-static) responsivity of the sensor to fibre displacement. It reaching its maximum value when the fibre's shadow overlapped-and its centre was aligned with-just one vertical edge of the photodiode detector. This stage of the calibration process has been reported elsewhere [12]. Following such a calibration procedure, any additional low-level vibration of the vertical shadow, at a given $V M$ frequency, would sweep the shadow back-and-forth across the face of the photodiode detector - between effectively two fixed (fibre) positions; and, as a result of the calibration, the $D C$ (quasi-static) responsivity would have been established over this span. Unfortunately, the response of the attendant amplifier to $A C$ photocurrents was different from its response to the (change in) $D C$ photocurrent, over the same excursion of fibre position; but if the ratio of the amplifier's $A C$ to $D C$ responses could be found, then the detection system's $A C$ responsivity to shadow displacement could be inferred from its measured $D C$ responsivity. It is the determination of this ratio that is reported here.

However, in order to accomplish this, a method needed to be found for illuminating the photodiode detector with a NIR beam having both a steady level of mean irradiance, and an accurately known (relative) depth of sinusoidally modulated irradiance-at a controlled frequency. The mean level of irradiance would generate the amplifier's $D C$ output signal, whilst the sinusoidal modulation would generate the corresponding ' $V M$ ' (AC) output, so that the ratio of these signals - and so their relative responsivities-could be linked. The approach that was taken, together with the results obtained, are reported below. It turned out that the VM $(A C)$ displacement responsivity would be found to lie (typically) in the Megavolt(s) per metre of fibre displacement range.

\section{The prototype Violin-Mode amplifier}

\subsection{Separating the photocurrents: the amplifier's DC and AC outputs}

The circuit diagram of the prototype Violin-Mode amplifier is shown in Figure 1, a subsequent dual-input version being covered in [10, and references therein]. Here, a Hamamatsu S2551 photodiode has a small reverse bias ( $\sim .5 \mathrm{~V})$ due the self-biasing action of the JFET (BF862) transistor, at a drain current $1.1 \mathrm{~mA}$. The source-follower action of the JFET transistor prevented $A C$ voltages from being impressed across the photodiode, and its connecting cable, thereby reducing considerably any 'noise-gain peaking' in the amplifier's AC response [10]. In the Figure, the photodiode detector is shown being illuminated from the left by a NIR beam of constant mean intensity, this beam having an additional small-amplitude sinusoidal modulation of its intensity, at a known frequency. Thus, if the reverse photocurrent generated in the photodiode by the beam is $I_{\text {photo, }}$ as indicated, then $I_{\text {photo }}=I_{D C}+i$, where $I_{D C}$ is a steady $D C$ photocurrent, and $i$ is an incremental $A C$ photocurrent, due to the modulation, superimposed onto the steady $D C$ current flow. 


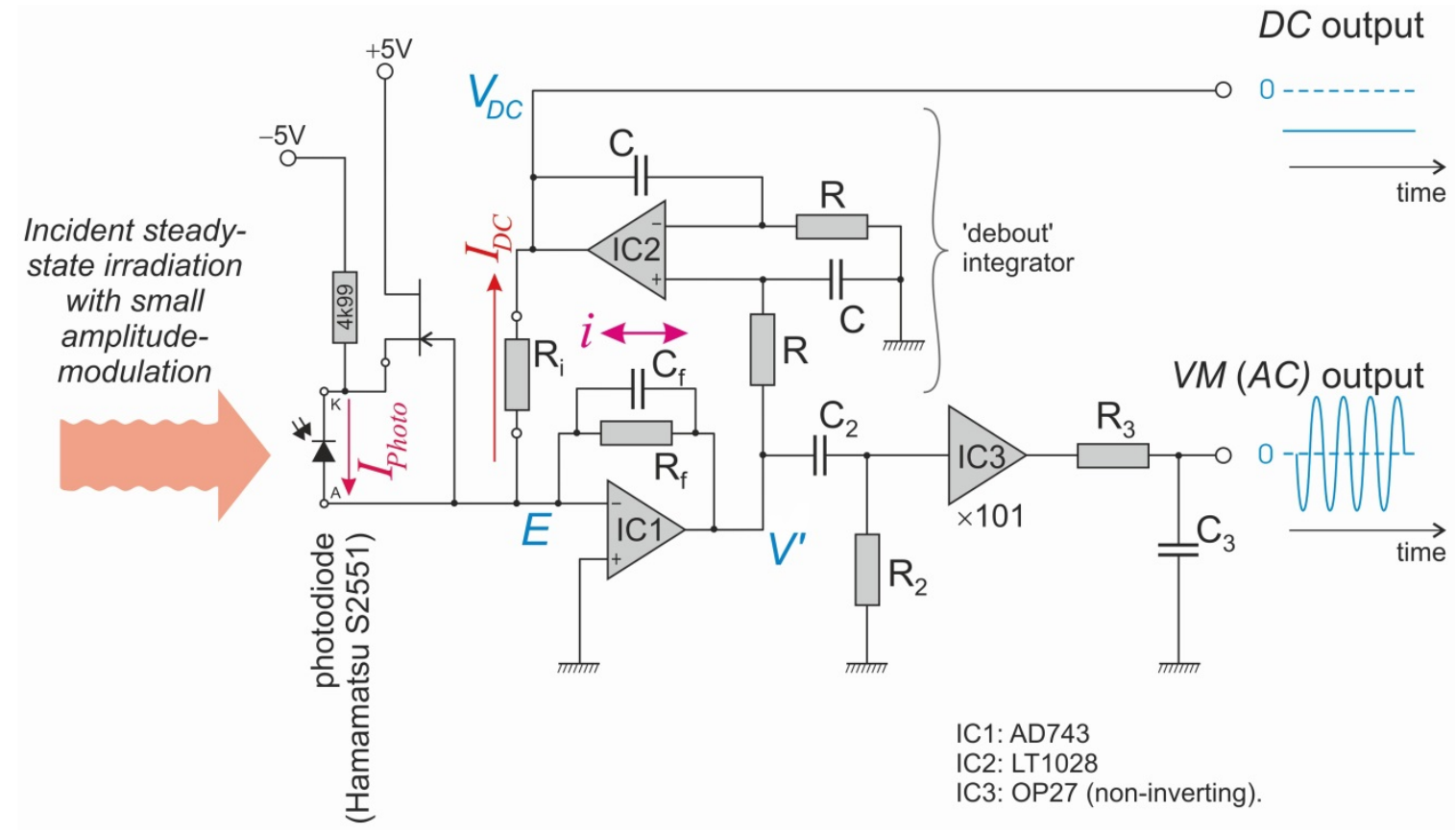

Figure 1. Circuit diagram of the prototype VM amplifier ( $\pm 15 \mathrm{~V}$ supply omitted). In the Figure, Near InfraRed radiation, incident from the left onto the photodiode, consists of a steady flux with a small superimposed intensity modulation. The illumination of the reverse-biased photodiode causes a photocurrent $I_{\text {photo }}$ to flow through it, as indicated, where $I_{\text {photo }}=I_{D C}+i$. Here, $I_{D C}$ is a steady $D C$ photocurrent, and $i$ is the incremental $A C$ photocurrent due to the modulation. The 'debout' (French for 'upright,' or non-inverting) integrator in the circuit, taking as its input the voltage $V$ ', forces these two photocurrents to follow the different transduction paths indicated in the Figure. This action is explained in the text. Separately, the JFET transistor in the circuit mitigates any 'noise-gain peaking' due to the capacitance of the photodiode (please refer to the text). In the Figure: $R=100 \mathrm{k}, \mathrm{C}=100 \mathrm{n}, \mathrm{R}_{\mathrm{i}}=120 \mathrm{k}, \mathrm{R}_{\mathrm{f}}=1 \mathrm{M} 2, \mathrm{C}_{\mathrm{f}}=10 \mathrm{p} 7, \mathrm{C}_{2}=100 \mathrm{n}, \mathrm{R}_{2}=10 \mathrm{k}, \mathrm{R}_{3}=3 \mathrm{k}$, and $\mathrm{C}_{3}=1000 \mathrm{p}$.

In Figure 1, IC1 and IC2 are op-amps, and IC3 is a simple non-inverting (op-amp based) amplifier, with an $A C$ voltage gain of $\times 101$; and the negative, integrator, feedback in the circuit causes the $D C$ photocurrent, $I_{D C}$, and the $A C$ (Violin-Mode) photocurrent, $i$, to follow the different paths indicated. Consequently, $V_{D C}=-I_{D C} \mathrm{R}_{\mathrm{i}}$ (always a negative voltage), and $-\mathrm{R}_{\mathrm{i}}\left(\mathrm{R}_{\mathrm{i}}=120 \mathrm{k} \Omega\right)$ is seen to be the $D C$ transimpedance gain of the amplifier.

In a similar way, $-R_{f}\left(R_{f}=1.2 \mathrm{M} \Omega\right)$ is seen to be the $A C$ transimpedance gain of the amplifier, if taken just to the output of IC1 (ignoring for the moment the effect of $C_{f}$ ). However, this output voltage is high-pass filtered by $\mathrm{C}_{2}-\mathrm{R}_{2}$, before being post-amplified by IC3, which is an $A C$ amplifier, having a non-inverting voltage gain of $\times 101$. The output of IC3 is then low-pass filtered by $\mathrm{R}_{3}-\mathrm{C}_{3}$, so as to roll-off the $A C$ signal gain more strongly above frequencies $\sim 12 \mathrm{kHz}$, where there are no $V M$ signals of interest. Thus, the $A C$ transimpedance gain of the amplifier is seen to be effectively $-101 R_{f}$, mid-band (i.e., at $1.71 \mathrm{kHz}$ ), or $121.2 \mathrm{M} \Omega$ —-ignoring the sign.

In summary, the theoretical $D C$ transimpedance gain of this amplifier was $(-) 120 \mathrm{k} \Omega$, whilst the corresponding $A C$ transimpedance gain was (nominally) $(-) 121.2 \mathrm{M} \Omega-$ midband. Therefore, the ratio of its $A C$ to $D C$ responses to the same change in photocurrent was set nominally at 1010, mid-band. In fact, the amplifier's fairly narrow bandwidth resulted in the passband's plateau peaking — theoretically-at the slightly lower mid-band value of 1004. 


\subsection{The prototype VM amplifier's output frequency responses}

The prototype VM amplifier which was constructed for this work, and whose circuit is shown in Figure 1, can be analysed straightforwardly to yield, in terms of the complex frequency s, a transimpedance relationship at its $D C$ output of the form

$$
\frac{V_{D C}}{I_{D C}}=-\frac{\mathrm{R}_{\mathrm{f}}}{\left(\mathrm{s}^{2} \mathrm{CRC} \mathrm{f}_{\mathrm{f}} \mathrm{R}_{\mathrm{f}}+\mathrm{sCR}+\mathrm{R}_{\mathrm{f}} / \mathrm{R}_{\mathrm{i}}\right)}
$$

Clearly, for a strictly $D C$ component of photocurrent (i.e., $s=0$ ) Equation 1 reduces to the simple expression $V_{D C}=-I_{D C} \mathrm{R}_{\mathrm{i}}$, as mentioned in §2.1. For the component values used (given in the caption of Figure 1), the expression in equation 1 is effectively that of a lowpass response, with a dominant pole at $161 \mathrm{~Hz}$ (and a second, $\mathrm{HF}$, pole at $12.2 \mathrm{kHz}$ ). In a similar fashion, the ratio of the amplifier's $A C$ response to a VM signal photocurrent, to its $D C$ response to a steady quiescent photocurrent, i.e., the amplifier's ratio of transimpedance gains, can be found to be

$$
\frac{V_{A C}}{V_{D C}(\mathrm{~s}=0)}=\frac{A\left(\mathrm{R}_{\mathrm{f}} / \mathrm{R}_{\mathrm{i}}\right) \mathrm{s}^{2} \mathrm{CRC}_{2} \mathrm{R}_{2}}{\left(\mathrm{~s}^{2} \mathrm{CRC} \mathrm{R}_{\mathrm{f}} \mathrm{R}_{\mathrm{f}}+\mathrm{sCR}+\mathrm{R}_{\mathrm{f}} / \mathrm{R}_{\mathrm{i}}\right)\left(\mathrm{sC}_{2} \mathrm{R}_{2}+1\right)\left(\mathrm{sC}_{3} \mathrm{R}_{3}+1\right)}
$$

The magnitude of Equation 2 is plotted as the dashed line in Figure 5 as a function of the frequency $f$ (in $\mathrm{Hz}$ ), using $A=101$, and using the component values given in the Figure 1 caption. Here, the value of the small capacitor $\mathrm{C}_{f}$ (nominally $10 \mathrm{pF}$ ) was used as a fitting parameter (resulting in $\mathrm{C}_{\mathrm{f}}=10.7 \mathrm{pF}$ ).

\subsection{Practical Near InfraRed (NIR) gain calibration}

Equation 2 expresses the relative sensitivity of the amplifier's $A C$ and $D C$ outputs to changes in photocurrent due to the same displacement of a fibre's shadow falling across (the edge of) the photodiode detector: in the one case a change occurring at a frequency $\mathrm{s}(=\mathrm{j} \omega$, for a sinusoidal modulation at angular frequency $\omega$, where $\mathrm{j}=\sqrt{-1}$, and $\omega=2 \pi f)$; and in the other a change occurring from one steady value to another. It is this expression of relative dependency which motivated the practical calibration of the $V M(A C)$ displacement responsivity, at a given modulation frequency, $f$.

A calibration system was therefore designed and built in order to measure the ratio of the $A C$ to $D C$ responses of an S2551 photodiode, connected to the prototype amplifier. A short $(<80 \mathrm{~mm})$ miniature coaxial lead was used to make this electrical connection, but, in order to simulate the capacitance of a coaxial cable $6+\mathrm{m}$ long (as might be used in a Gravitational Wave Detector environment), a $680 \mathrm{pF}$ capacitor was placed in parallel with the photodiode.

\section{A calibration system for both $A C$ and $D C$ responsivities}

\subsection{The calibration source}

The calibration system needed to irradiate the amplifier's single photodiode detector with a very low intensity beam from a NIR LED, the beam consisting of a steady component of fixed intensity, plus a small sinusoidal intensity-modulation on top of this, at a known frequency, $f$. Figure 2 shows the circuit diagram of the calibration source for the photodiode/amplifier that was constructed for this work. The NIR beam's modulationcontrolling circuit was based on a published article which described the control of an IPL10530KAL device [13]. In this work, however, separate devices were used: a discrete BPW34S photodiode (PD) was mounted off-axis at a distance of approximately $15 \mathrm{~mm}$ 


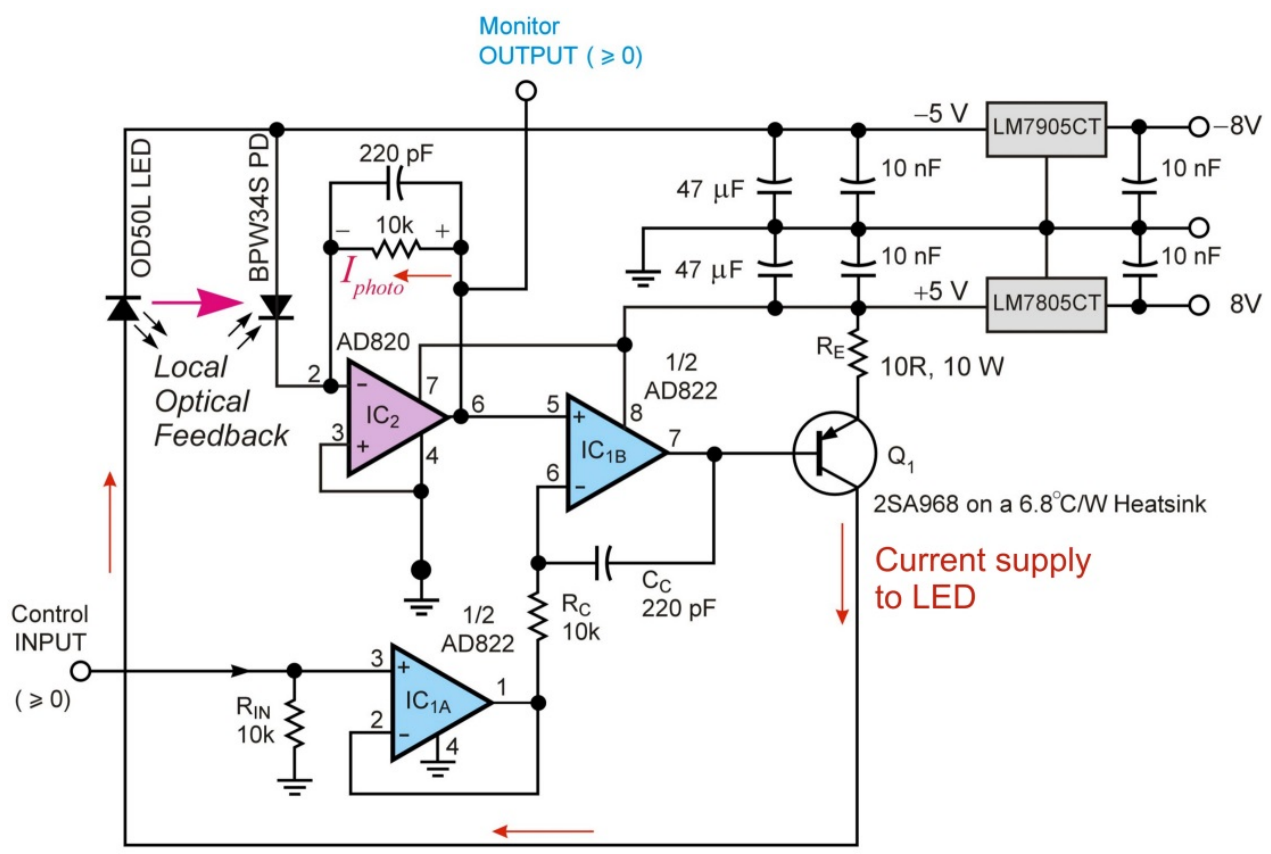

Figure 2. InfraRed modulation circuit derived from an original article by Lukasz Sliwczynskia and Marcin Lipinski [13]. Two voltage regulators supply the $\pm 5 \mathrm{~V}$ rails for the Near InfraRed intensity controlling and modulation circuit. Via local, optical, feedback, the NIR beam emitted by the OD50L LED was a precise analogue in its intensity of the voltage at the 'Control INPUT'-for both the $D C$ level and for any $A C$ modulation superimposed on top of this level, i.e., for the sum of any such voltages, provided the sum was $\geq 0$.

from the lens of a high-power OD50L, NIR LED, emitter $(\lambda=880 \mathrm{~nm})$, the PD being located at an angle of approximately $5^{\circ}$ from the LED's beam-axis. The PD monitored the emitter's output beam intensity, whilst an AD820 op-amp, together with its indicated feedback components, acted in a transimpedance rôle.

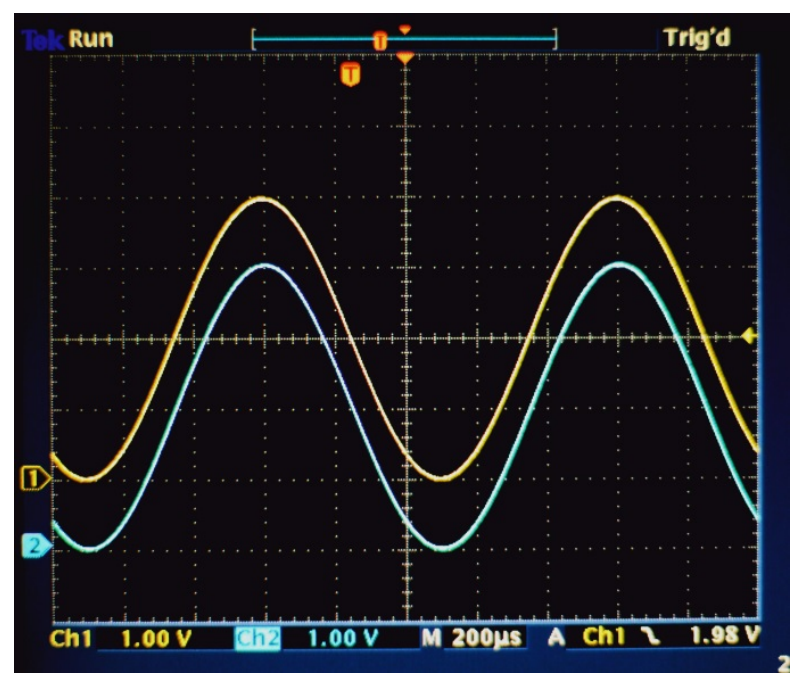

Figure 3. Example of the optical feedback control of emitted intensity. In this example a very high level of relative modulation was used. Upper trace (yellow): Control INPUT to the circuit of Figure 2, showing a 'command' voltage in the form of a $1 \mathrm{kHz}, 4.0$ volt peak-peak, modulation, about a steady 2.0 volt $D C$ level. Lower trace (blue): Monitor OUTPUT (offset down the screen by approximately 1 volt). Here, local, optical, negative feedback between the OD50L LED and the local BPW34S silicon photodiode has forced the intensity of the beam emitted by the NIR LED to be a close analogue of the command voltage-even under these rather extreme conditions (+5 volt supply to the circuit, and total input control voltage descending to zero volts). 
The Control INPUT voltage to the circuit shown in Figure 2 consisted of a positive $D C$ level, about which a (generally) small-amplitude sinusoidal $A C$ modulation was added, this modulation being at a fixed frequency, $f$, in the range $1 \mathrm{~Hz}-300 \mathrm{kHz}$. A $D C$ current of typically $\sim 200 \mathrm{~mA}$ was generated by the $D C$ voltage (typically, $\sim 2 \mathrm{~V}$ ) applied to this Control input. It was sourced by the pnp transistor, $\mathrm{Q}_{1}$, which powered the OD50L emitter. Local, optical, feedback via the BPW34S photodiode then slaved the LED's emitted output intensity to be a very precise analogue of the control input voltage (both $D C$ level and $A C$ modulation) - and this was verified at the circuit's Monitor output, where, under correct operation, Monitor OUTPUT voltage $=$ Control INPUT voltage. Figure 3 shows an example of this action. In this way, the intensity of the NIR beam emitted by the LED became a very close analogue of the Controlling input voltage - for both the $D C$ level and the $A C$ modulation.

\subsection{AC and DC relative gain measurements}

Figure 4 shows schematically a modulated NIR beam, coming from the OD50L LED, being used to illuminate the prototype amplifier's photodiode, via a pinhole in a mask. The LED was positioned with the front of its lens approximately $35 \mathrm{~mm}$ from the mask, and with its beam incident normally onto the mask. The pinhole was $0.35 \mathrm{~mm}$ in diameter. On the Control INPUT side of the source, as shown in Figure 2, both the $D C$ and modulation (peak-peak) levels were noted for the particular modulation frequency being used. These waveforms then were checked for fidelity (i.e., proper feedback control) at the Monitor OUTPUT of the source. The resulting $A C$ and $D C$ output signals from the prototype amplifier also were measured at this time. The ratio of the amplifier's $A C / D C$ gain then was computed from these four measured signals, for that particular modulation frequency-as described in the following example (illustrated in Figure 4).

The three graphs shown in Figure 4 are actual oscilloscope screen shots taken during a measurement: at the lower right of the Figure the Control input is seen to consist of a $D C$ level = 2.151 V, plus a small sinusoidal modulation [58.0 $\mathrm{mV}$ (peak-peak), at $1 \mathrm{kHz}$ ]. Here, the Monitor output signal was indistinguishable from that at the Control input-over the frequency range $1 \mathrm{~Hz} \leq \mathrm{f} \leq 300 \mathrm{kHz}$; in the lower left screen shot the amplifier's $D C$ output $=-1.023 \mathrm{~V}$; and in the top screen shot the amplifier's $A C$ output $=27.33 \mathrm{~V}$ (peak-peak)-in anti-phase with the input modulation at $1 \mathrm{kHz}$, as expected. Therefore, in this example the measured $A C / D C$ gain ratio $=(27.33 / 58.0 \mathrm{e}-3) /(1.023 / 2.151)=991( \pm 4)$, at $1 \mathrm{kHz}$.

This procedure was repeated for modulation frequencies in the range $1 \mathrm{~Hz}-300 \mathrm{kHz}$, although, towards the lower end of this frequency range, the amplitude of modulation had to be increased — so that the $A C$ response might become more easily measurable.

The results of such measurements as a function of the modulation frequency $f$ are shown in Figure 5. Clearly, the measurements followed the theoretical expectation very well up to $\sim 100 \mathrm{kHz}$, i.e., well above the required $V M$ bandwidth, which extended from $500 \mathrm{~Hz}-$ $5 \mathrm{kHz}$. Indeed, Equation 2 gives the theoretical $A C / D C$ gain ratio to be 927.1 at $500 \mathrm{~Hz}$, and 994.2 at $1 \mathrm{kHz}$, whereas the measured values, using the method shown in Figure 4, gave this same ratio as being $922.5 \pm 4$ at $500 \mathrm{~Hz}$, and $991 \pm 4$ at $1 \mathrm{kHz}$.

For the numerical example illustrated in Figure 4, the $A C$ responsivity $=991 \times$ the $D C$ responsivity (measured separately, as explained in §1; and in [12]), at $1 \mathrm{kHz}$.

In fact, a maximum $D C$ responsivity of $1.26 \mathrm{kV} . \mathrm{m}^{-1}$ was measured using the prototype photodiode sensor and amplifier discussed here. Therefore, the measured $A C / D C$ transimpedance gain ratio of 922.5 for this sensor, at $500 \mathrm{~Hz}$, translated into a maximum VM $(A C)$ responsivity of $(1.16 \pm 0.05) \mathrm{MVm}^{-1}$, at that frequency. 


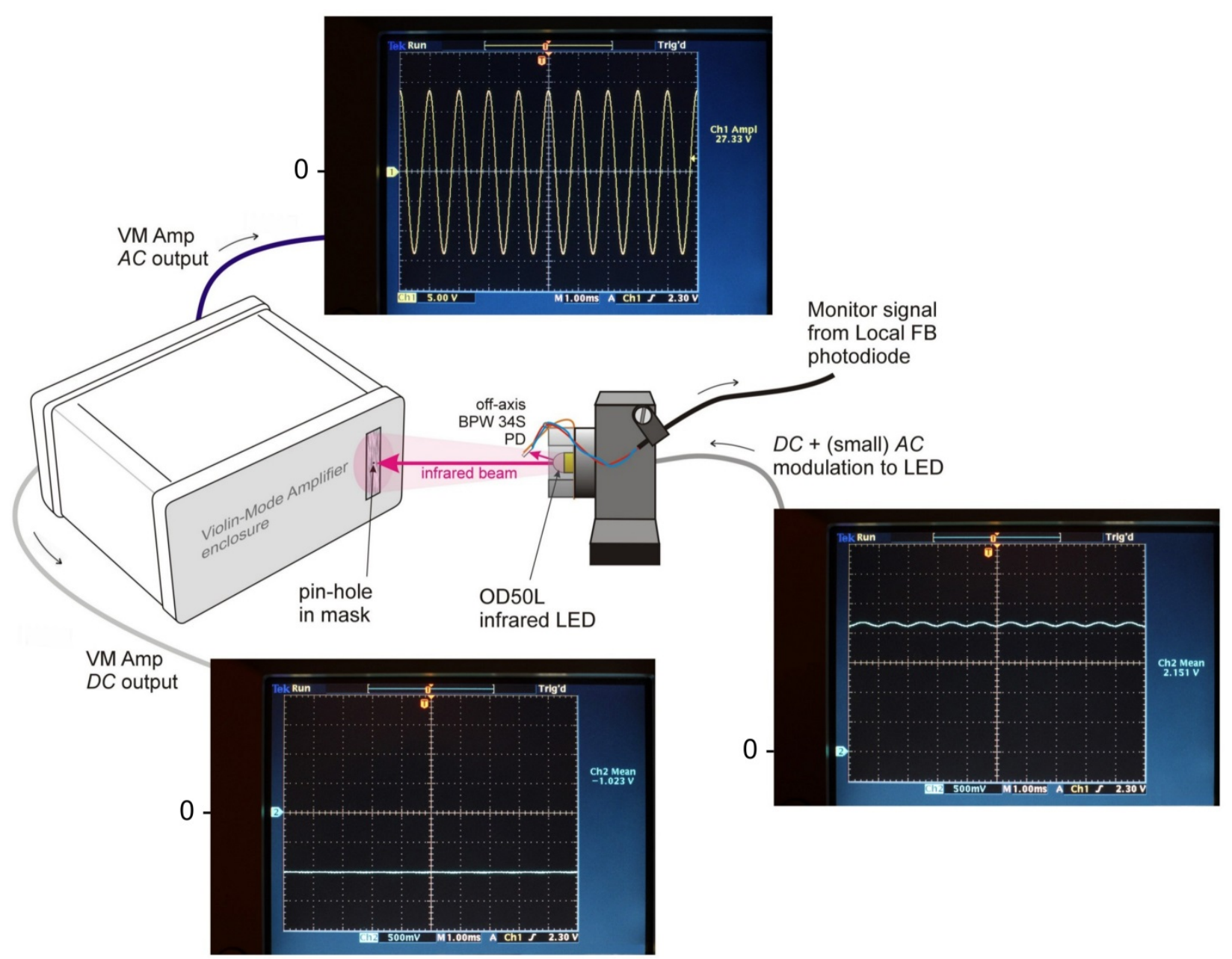

Figure 4. Schematic diagram showing a measurement of the $A C / D C$ gain ratio of the photodiode/prototype amplifier at a particular frequency, $f$ (here, $f=1 \mathrm{kHz}$ ). The infrared beam from the OD50L infrared LED was incident onto the amplifier's S2551 photodiode via a pin-hole in an aluminium foil mask. The three graphs are actual oscilloscope screen shots: on the right the Control Input $=2.151 \mathrm{~V}+(58.0 \mathrm{mV}$ p-p, $1 \mathrm{kHz}$, modulation); in the lower left screen shot the amplifier's $D C$ output $=-1.023 \mathrm{~V}$; and in the top screen shot the amplifier's $A C$ output $=27.33 \mathrm{~V}_{\mathrm{p}-\mathrm{p}}$, this being in anti-phase with the input modulation at $1 \mathrm{kHz}$, as expected. The $D C$ zero voltage levels have been emphasized in each of the screen shots. Please refer to the text for the $A C / D C$ gain ratio calculation.

The measured ratio of the $A C / D C(s=0)$ transimpedance gains for the prototype amplifier/ photodiode shadow detector, shown in Figure 5, clearly followed the theoretical curve (indicated by the dashed line in the Figure) very well indeed, up to $\sim 100 \mathrm{kHz}$. Evidently, the amplifier was functioning as anticipated, from the theory given in $\S 1$ and $\S 2$. One small feature of the measurements of the $A C / D C$ gain ratio that was noticed, however, was that between its two $-3 \mathrm{~dB}$ points (give in the caption to Figure 5), where this ratio approached a value of 1000 , the measured values tended to lie slightly below $(<1 \%$ below) the theoretical expectations. It could be argued that only $1 \%$ tolerance resistors had been used in constructing the amplifier, and so the agreement was unexpectedly close, anyway; but then this shortfall did not extend either to lower, or to higher frequencies, $f$ (for $f<100 \mathrm{kHz}$, that is). An alternative explanation might be that in this frequency regime the $A C$ output signals were typically $\sim 27$ volts (peak-peak). No obvious distortion was seen in such cases, but this 
level of excursion lies close to the maximum voltage swing of the OP27E op-amp's outputwith a $\pm 15 \mathrm{~V}$ supply. In retrospect, it might have been better to have limited such voltage swings to $\sim 25$ volts (peak-peak), say. The fall-off in the $A C / D C$ gain ratio above $\sim 100 \mathrm{kHz}$, however, was due to the effective bandwidth of the $A C$ amplifier built around IC3, which was expected to be only $\sim 80 \mathrm{kHz}$. This additional roll-off in response towards higher frequencies

\subsection{The AC/DC ratio of transimpedance gains, measured as a function of frequency}

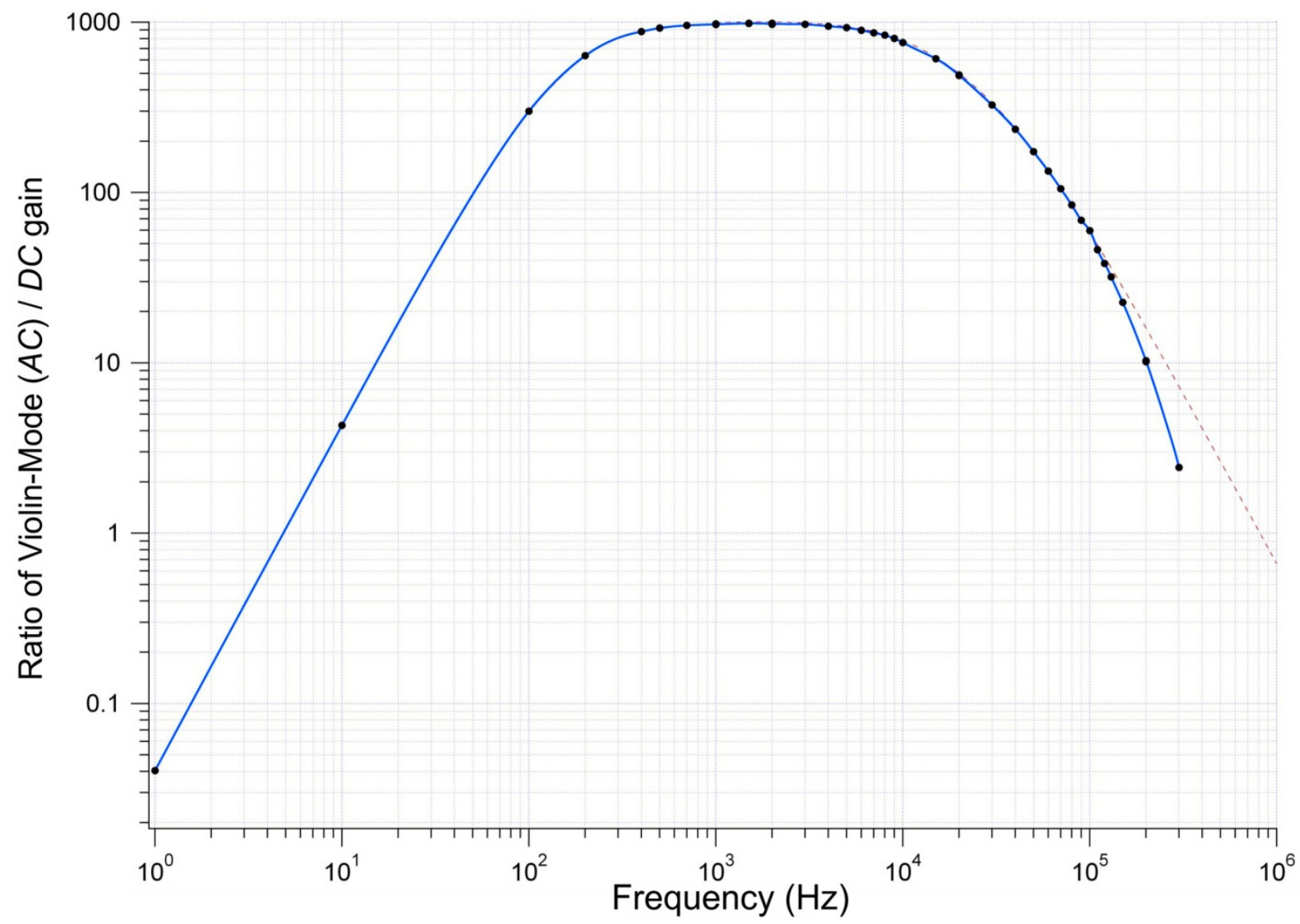

Figure 5. Measured ratio of the $A C / D C(s=0)$ transimpedance gains for the prototype amplifier/photodiode, as a function of the modulation frequency, $f$, using the calibration apparatus shown in Figures 2 and 4 . The solid data points are measurements made using the technique shown in Figure 4, the (blue) full-line through these data being simply a guide to the eye. The dashed line running from $1 \mathrm{~Hz}-1 \mathrm{MHz}$ (largely obscured) is the magnitude of the theoretical gain ratio, given by Equation 2. For these measurements an additional $680 \mathrm{pF}$ capacitor was placed in parallel with the single Hamamatsu S2551 photodiode, in order to simulate the capacitance of $6+\mathrm{m}$ of co-axial cable running between the photodiode and the amplifier (please refer to the text). The $-3 \mathrm{~dB}$ frequencies for the ratio of the $A C / D C$ gains were found here to be $241 \mathrm{~Hz}$, and $12074 \mathrm{~Hz}$. The phase response gave a $33^{\circ}$ phase-lead at a frequency of at $500 \mathrm{~Hz}$, moving to a phase lag of $24^{\circ}$ at $5 \mathrm{kHz}$.

was seen as being positively beneficial, however-from the point of view of reducing the overall noise-bandwidth of the amplifier.

\section{Conclusions}

A bench measurement of the shadow-sensor's quasi-static responsivity to suspension fibre displacement (or $D C$ responsivity), clearly allows the more valuable $A C$ responsivity to be inferred-with a high degree of confidence, given the agreement with theory - at any specified VM frequency; but the use of a transimpedance amplifier having two separate outputs ( $D C$ and $A C$ ) is then a sine qua non. Moreover, the ratio of $A C / D C$ transimpedance gains for the amplifier then has to be found, as shown in Figure 5, and, in order to make that measurement, the modulated illuminating source, as described here in $\S 3.1$ and §3.2, has 
turned out to be indispensable. In contrast, trying to vibrate the entire fibre, bodily, within the illuminating beam, as an isolated entity (having no physical supports, and no excited vibrational modes), and with an accurately-known amplitude of $1.00 \mu \mathrm{m}$, say, across the whole frequency range, was never an option.

The single photodiode detector / prototype transimpedance amplifier discussed here was developed, eventually, into the split-photodiode / dual-input transimpedance amplifier, discussed in $[9,11]$; and, for comparison, the $D C$ responsivity of this later development was measured at $10.7 \mathrm{kVm}^{-1}$, averaged over its four sensors, compared with the $1.26 \mathrm{kV} . \mathrm{m}^{-1}$ reported here. The effective $A C / D C$ gain ratio for the dual-input amplifier was $904 \pm 4$ at $500 \mathrm{~Hz}$ - determined by placing the pinhole mask over each detector element, separatelyleading to a high (average) VM responsivity of $9.65 \mathrm{MVm}^{-1}$, at this frequency.

At the time of writing neither the Violin-Mode amplifier and sensor system described here, nor its successor $[8,9,11,14]$, has been adopted for aLIGO, and, indeed, the need for separate VM sensing and damping has not yet been demonstrated. The current baseline solution is to use aLIGO's Arm Length Stabilization system as a VM sensor / damper [15]. In fact, the issue of vacuum compatibility remains unresolved for the $V M$ sensor described here, and its successor, because the Hamamatsu photodiodes used for the detector elements had been encapsulated, using an unknown epoxy. However, were it to become necessary, the issue of the epoxy for the photodiodes from this, or another, manufacturer probably could be resolved, and the LEDs and other components used are likely to prove vacuum compliant, or have vacuum-compliant alternatives.

\section{Acknowledgements}

We thank the IGR, University of Glasgow, Scotland, UK, particularly Ken Strain and Angus Bell, for their encouragement with this work. Thanks go to Norna Robertson and Calum Torrie of Caltech and the IGR, Alberto Vecchio of the University of Birmingham, and Justin Greenhalgh of the CCLRC (RAL), for their oversight of, and assistance with, this work. We are grateful to the staff of the Physics Department's Electronics Workshop, and the Science Faculty's Mechanical Workshop, at the University of Strathclyde, for their careful construction of most of the component parts used in this work, and we would also like to thank the company Bar Knight Precision Engineers Ltd. of Clydebank, Scotland, for their very good, and timely, machining work. Finally, we are grateful for the support of grant STFC PP/F00110X/1, which sustained this work.

\section{References}

[1] Harry G M (for the LIGO Scientific Collaboration) 2010 Advanced LIGO: the next generation of gravitational wave detectors. Class. Quantum Grav. 27084006 (12pp).

[2] Raab F J et al 2004 Overview of LIGO Instrumentation Proceedings of SPIE $\mathbf{5 5 0 0}$ 11-24 (29 Sept.).

[3] Abbott B P et al 2009 LIGO: The Laser Interferometer Gravitational-Wave Observatory Rep. Prog. Phys. 72076901.

[4] Aston S M et al 2012 Update on quadruple suspension design for Advanced LIGO Class. Quantum Grav. 29235004 (25pp).

[5] Heptonstall A et al 2011 Invited Article: $\mathrm{CO}_{2}$ laser production of fused silica fibers for use in interferometric gravitational wave detector mirror suspensions Rev. Sci. Instrum. 82011301 1-9.

[6] Cumming A V et al 2012 Design and development of the advanced LIGO monolithic fused silica suspension Class. Quantum Grav. 29035003 (18pp). 
[7] Hamamatsu S2551 photodiode: http://www.hamamatsu.com/.

[8] Lockerbie N A and Tokmakov K V and Strain K A 2014 A source of illumination for low-noise 'Violin-Mode' shadow sensors, intended for use in interferometric gravitational wave detectors Meas. Sci. Technol. 25,12, 12 p., 125111; http://dx.doi.org/10.1088/0957-0233/25/12/125111.

[9] Lockerbie N A and Tokmakov K V 2014 A 'Violin-Mode' shadow sensor for interferometric gravitational wave detectors Meas. Sci. Technol. 25, 12, 12 p., 125110; http://dx.doi.org/10.1088/0957-0233/25/12/125110.

[10] Dmitriev A et al 2010 Controlled damping of high-Q violin modes in fused silica suspension fibers. Class. Quantum Grav. 27025009 (8pp).

[11] Lockerbie N A and Tokmakov K V 2014 A low-noise transimpedance amplifier for the detection of 'Violin-Mode' resonances in advanced LIGO suspensions Rev. Sci. Instrum., 85, 11, 8 p., 114705; http://dx.doi.org/10.1063/1.4900955.

[12] Lockerbie N A and Tokmakov K V 2014 Quasi-static displacement calibration system for a 'Violin-Mode' shadow-sensor in Advanced LIGO suspensions Rev. Sci. Instrum., 85, 10, 12 p., 105003; http://dx.doi.org/10.1063/1.4900955.

[13] Circuit derived from an article by Lukasz Sliwczynskia and Marcin Lipinski, Institute of Electronics, Krakow, Poland, EDN, 1 September,1998, p92.

[14] Lockerbie N A et al 2011 First results from the 'Violin-Mode' tests on an advanced LIGO suspension, at MIT. Class. Quantum Grav. 28245001 (12pp).

[15] Instrument Science White Paper 2012 LIGO-T1200199-v2, p71 https://dcc.ligo.org. 